\title{
Is MR-guided High-intensity Focused Ultrasound a Feasible Treatment Modality for Desmoid Tumors?
}

\author{
Raffi S. Avedian MD, Rachelle Bitton PhD, Garry Gold MD, \\ Kim Butts-Pauly PhD, Pejman Ghanouni MD, PhD
}

Published online: 4 June 2015

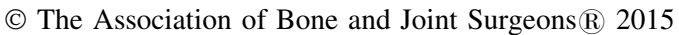

\begin{abstract}
Background MR-guided high-intensity focused ultrasound is a noninvasive treatment modality that uses focused ultrasound waves to thermally ablate tumors within the human body while minimizing side effects to surrounding healthy tissues. This technology is FDAapproved for certain tumors and has potential to be a noninvasive treatment option for extremity soft tissue tumors. Development of treatment modalities that achieve tumor control, decrease morbidity, or both might be of great benefit for patients. We wanted to assess the potential
\end{abstract}

One of the authors (GG) has received research support funding from General Electric (Medical Systems, Milwaukee, WI, USA) not related to this study. The National Institutes of Health provided funding support for this project, PO1 CA159992 (KB-P, RSA, PG, GG). All ICMJE Conflict of Interest Forms for authors and Clinical Orthopaedics and Related Research ${ }^{\mathbb{R}}$ editors and board members are on file with the publication and can be viewed on request.

Clinical Orthopaedics and Related Research ${ }^{\circledR}$ neither advocates nor endorses the use of any treatment, drug, or device. Readers are encouraged to always seek additional information, including FDAapproval status, of any drug or device prior to clinical use.

Each author certifies that his or her institution approved the human protocol for this investigation, that all investigations were conducted in conformity with ethical principles of research, and that informed consent for participation in the study was obtained.

\footnotetext{
R. S. Avedian $(\square)$

Department of Orthopaedic Surgery, Stanford University, 450 Broadway Street, MC 6342, Redwood City, CA 94063, USA e-mail: ravedian@stanford.edu

R. Bitton

Stanford University, Stanford, CA, USA

G. Gold, K. Butts-Pauly, P. Ghanouni

Department of Radiology, Stanford University, Stanford, CA, USA
}

use of this technology in the treatment of extremity desmoid tumors.

Questions/purposes (1) Can we use MR-guided highintensity focused ultrasound to accurately ablate a predetermined target volume within a human cadaver extremity? (2) Does MR-guided high-intensity focused ultrasound treatment stop progression and/or cause regression of extremity desmoid tumors?

Methods Simulated tumor volumes in four human cadavers, created by using plastic markers, were ablated using a commercially available focused ultrasound system. Accuracy was determined in accordance with the International Organization of Standards location error by measuring the farthest distance between the ablated tissue and the plane corresponding to the target. Between 2012 and 2014, we treated nine patients with desmoid tumors using focused ultrasound ablation. Indications for this were tumor-related symptoms or failure of conventional treatment. Of those, five of them were available for MRI followup at 12 months or longer (mean, 18.2 months; range, 12-23 months). The radiographic and clinical outcomes of five patients who had desmoid tumors treated with focused ultrasound were prospectively recorded. Patients were assessed preoperatively with MRI and followed at routine intervals after treatment with MRI scans and clinical examination.

Results The ablation accuracy for the four cadaver extremities was $5 \mathrm{~mm}, 3 \mathrm{~mm}, 8 \mathrm{~mm}$, and $8 \mathrm{~mm}$. Four patients' tumors became smaller after treatment and one patient has slight progression at the time of last followup. The mean decrease in tumor size determined by MRI measurements was $36 \%$ (95\% confidence interval, $7 \%$ $66 \%$ ). No patient has received additional adjuvant systemic or local treatment. Treatment-related adverse events included first- and second-degree skin burns occurring in 
four patients, which were managed successfully without further surgery.

Conclusions This preliminary investigation provides some evidence that MR-guided high-intensity focused ultrasound may be a feasible treatment for desmoid tumors. It may also be of use for other soft tissue neoplasms in situations in which there are limited traditional treatment options such as recurrent sarcomas. Further investigation is necessary to better define the indications, efficacy, role, and long-term oncologic outcomes of focused ultrasound treatment.

Level of Evidence Level IV, therapeutic study.

\section{Introduction}

The treatment for extremity soft tissue tumors may involve surgery, radiation, chemotherapy, or a combination of these modalities depending on the grade and histology of the tumor, its size, location, and the patient's overall medical condition. Surgery is the mainstay of treatment for these neoplasms but it may result in substantial morbidity and compromise to quality of life. In some instances such as when treating desmoid tumors, the risks of a local recurrence and treatment-related morbidity are large enough that surgery may not be a feasible option [1, 4, 11, 13]. Development of treatment modalities that achieve tumor control, decrease morbidity, or both would therefore be of great benefit for patients.

MR-guided high-intensity focused ultrasound (MRgFUS) is a noninvasive treatment modality that can ablate target tissue or tumors within the body. MRgFUS is based on the physical properties of sound. When propagating through human tissue, sound energy causes friction within the tissues that leads to heating. In most circumstances, ultrasound results in very little energy deposition with no heating or damage to human tissue. However, when multiple sound waves converge to a single point, there is summation of energy that results in heating and thermal necrosis of the tissue. Because heating only occurs where the ultrasound waves converge, the surrounding tissue remains unaffected [8]. When treating tumors in a clinical setting, multiple treatment foci are targeted within the tumor in an overlapping manner such that at the completion of therapy, the sum of all the ablations results in complete tumor thermal necrosis. MR thermometry is done in real time during the MRgFUS treatment and allows direct visualization and temperature mapping of the target tissue. Postcontrast imaging can directly evaluate the extent of ablation within the tumor immediately after treatment. This technology is a noninvasive treatment modality that is FDA-approved for the treatment of uterine fibroids and bone metastasis. Several studies have demonstrated that focused ultrasound ablation can cause regression of uterine fibroids and alleviate pain from bone metastasis [12, 15, 20]. MRgFUS may also be useful in the treatment of extremity soft tissue tumors; however, investigations for this application have not to our knowledge been published. Desmoid tumors, also called aggressive fibromatosis, are a locally aggressive soft tissue tumor that have no metastatic potential. In some patients they are associated with familial adenomatous polyposis (Gardner's syndrome). In sporadic cases they are commonly located in the extremities, trunk, and abdominal wall and may be associated with pain and functional impairment. These tumors tend to recur even after treatment with complete surgical resection, chemotherapy, and/or radiotherapy and as such warrant investigation with new treatments such as MR-guided high-intensity focused ultrasound (MRgHIFU) that may be more effective or at least limit side effects of standard treatments $[10,17,18]$.

The purpose of this study therefore was to determine the feasibility of using MRgFUS for the treatment of extremity soft tissue tumors. We specifically asked the following study questions: (1) Can we use MR-guided high-intensity focused ultrasound to accurately ablate a predetermined target volume within a human cadaver extremity? (2) Does MR-guided high-intensity focused ultrasound treatment stop progression and/or cause regression of extremity desmoid tumors?

\section{Patients and Methods}

This study was performed in two parts. First, a cadaver model was created to determine accuracy and feasibility of using high-intensity focused ultrasound to treat a human tissue located in the extremities. Then, we evaluated radiographic tumor response to treatment in a small group of selected patients who were followed prospectively under a protocol and who underwent MRgFUS as part of their treatment for desmoid tumor.

We used a commercially available MRgFUS system to perform thermal ablation of simulated tumor volumes in four human cadaver extremities (Exablate 2000; Insightec, Haifa, Israel). The simulated tumor volumes were created by percutaneously inserting plastic intravenous catheters in a predetermined geometric pattern into the cadaver muscle (Fig. 1). A geometric pattern was chosen because this was thought to be easier to create and visualize on MR as well as assess on gross inspection compared with an oval shape. These catheters are visible on MRI and define the boundary of the simulated tumor. We conducted the ablations using standard settings of the MRgFUS device, which included a frequency range of $0.9 \mathrm{MHz}$ to $1.35 \mathrm{MHz}$ and energy deposition of $800 \mathrm{~J}$ to $2900 \mathrm{~J}$ per sonication. The goal of treatment was to ablate the simulated tumor volume. 


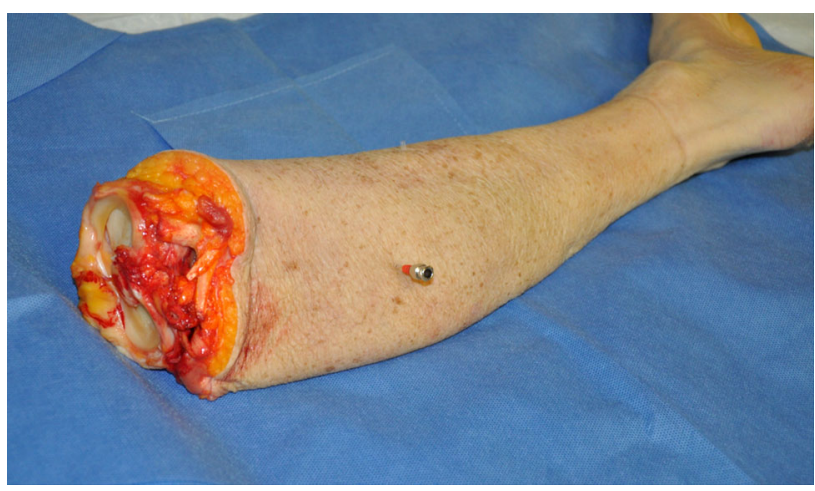

Fig. 1 Photograph showing a cadaver leg after insertion of an intravenous catheter used to create a target for MRgFUS ablation. The metal needle is removed before MR scanning.

On completion, we dissected each cadaver limb and measured the actual area of tissue ablation and compared that with the intended area of ablation as delineated by the fiducial catheters. Accuracy was determined by measuring the location error according to the International Organization of Standards [2]. The location error is defined as the longest vertical distance between the target ablation plane and the farthest point of actual ablation (Fig. 2). We examined each specimen for complications such as skin burns and unintended nerve or vascular burns. Because postcontrast sequences (or delayed MRI weeks later) are best at evaluating tumor ablation, we did not image the cadavers posttreatment because contrast administration in cadavers is obviously not possible. We found that there really was not a good way to assess ablation using MRI in cadavers.

After completion of the cadaver studies, we obtained approval from our institution's ethics committee to perform MRgFUS on patients with desmoid tumors. Between 2012 and 2014, we treated nine patients with desmoid tumors using focused ultrasound ablation. Indications for this were tumor-related symptoms or failure of conventional treatment. Of those, five of them were available for MRI followup at 12 months or longer (mean, 18.2 months; range, 12-23 months). Patients were included in this report if they had a minimum 12-month followup. No patients were lost to followup.

The clinical portion of our study included review for potential treatment by a multidisciplinary tumor board; patients were considered for MRgFUS treatment if they met the indications for treatment, which consisted of (1) biopsy-proven symptomatic extraabdominal fibromatosis; (2) morbidity of surgery considered unacceptable or progression of disease despite chemotherapy, surgery and/or radiation, or patient refusal of surgery, chemotherapy, and/ or radiation treatment; and (3) target volume within the tumor could be treated safely based on standard MRgFUS principles; these principles include but are not limited to:
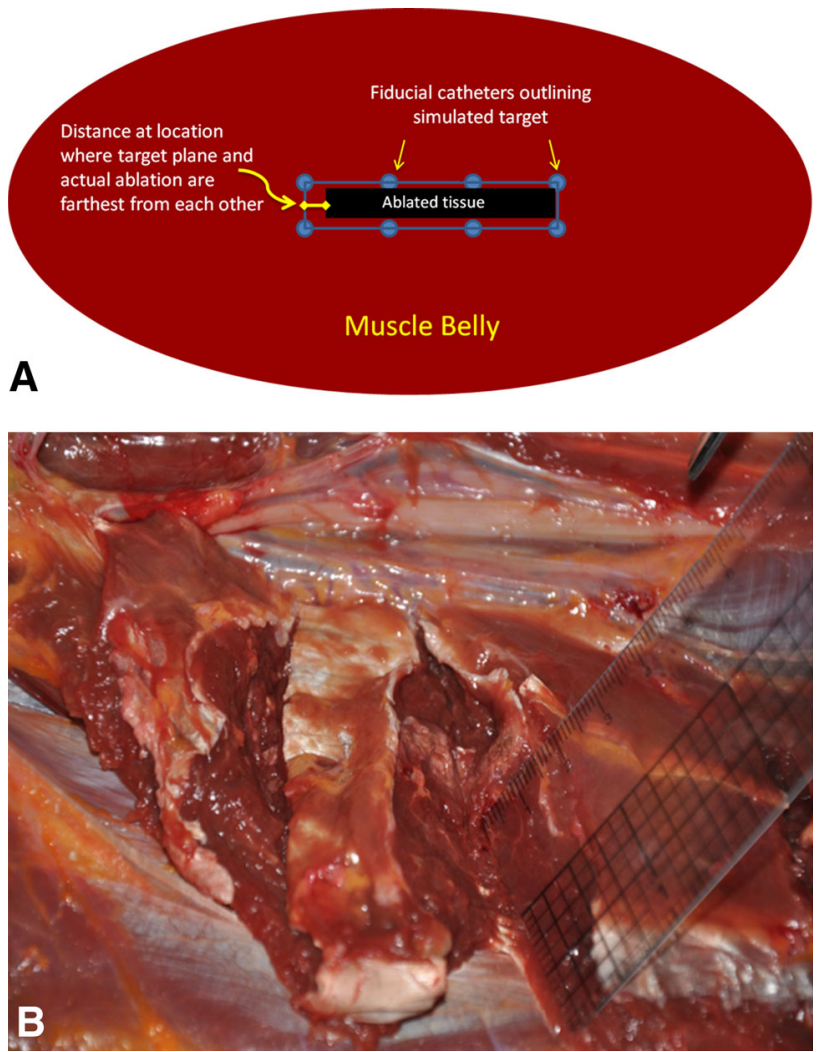

Fig. 2A-B Schematic (A) illustrating how the distance between the target plane and actual ablation region is measured; (B) photograph showing a cadaver leg dissection and appearance of ablated tissues (ruler), which appear lighter in color compared with normal muscle after MRgFUS.

ablating greater than $1 \mathrm{~cm}$ from critical anatomic structures such as skin, nerves, and major blood vessels, treating tissues that are amenable to MR thermometry, and not treating air-containing organs such as bowel because the air interface results in dangerous temperature spikes $[6,9,20]$. Five patients (three men, two women) with an average patient age of 28.2 years (range, 14-67 years) were treated. Four patients had progressive disease despite standard treatments, and one patient with symptomatic disease declined surgery, radiation, and systemic treatment (Table 1). All patients were extensively counseled and signed a comprehensive consent document. The study was approved by our institution's ethics committee. The mean followup was 18.2 months (range, 12-23 months).

Patients underwent general anesthesia $(\mathrm{n}=3)$, regional anesthesia $(\mathrm{n}=2)$, or a combination of general and regional anesthesia $(n=1)$ before the procedure. All procedures were planned in collaboration with an orthopaedic oncologist (RSA) and interventional radiologist (PG). An ExAblate MR-guided focused ultrasound system with an in-table transducer (InSightec, Tirat-Carmel, Israel) was used with a 70-cm bore 3-T MR system (Discovery 750w; 
Table 1. Patient characteristics

\begin{tabular}{|c|c|c|c|c|c|}
\hline $\begin{array}{l}\text { Patient } \\
\text { number }\end{array}$ & $\begin{array}{l}\text { Age (years), } \\
\text { sex }\end{array}$ & Location & Previous treatments & $\begin{array}{l}\text { Reason for high-intensity } \\
\text { focused ultrasound }\end{array}$ & $\begin{array}{l}\text { Number of high-intensity } \\
\text { focused ultrasound } \\
\text { treatments }\end{array}$ \\
\hline 1 & 28 , female & Chest wall & None & $\begin{array}{l}\text { Progression; patient did } \\
\text { not want other treatments }\end{array}$ & $\begin{array}{l}1 \text { (subsequent cryotherapy } \\
\text { to } 10 \% \text { of tumor) }\end{array}$ \\
\hline 2 & 18 , male & Popliteal fossa & $\begin{array}{l}\text { Surgery, radiation, } \\
\text { vinblastine, } \\
\text { methotrexate }\end{array}$ & $\begin{array}{l}\text { Not tolerating } \\
\text { chemotherapy, } \\
\text { progression }\end{array}$ & 2 \\
\hline 3 & 16 , male & Buttock & $\begin{array}{l}\text { Surgery, vinblastine, } \\
\text { methotrexate, } \\
\text { tamoxifen, sulindac }\end{array}$ & $\begin{array}{l}\text { Progression, not tolerating } \\
\text { chemotherapy }\end{array}$ & 3 \\
\hline 4 & 15 , female & Popliteal fossa & $\begin{array}{l}\text { Vinblastine, } \\
\text { methotrexate, Gleevec }\end{array}$ & Progression & 1 \\
\hline 5 & 66 , male & Posterior ankle & None & $\begin{array}{l}\text { Progression, symptomatic, } \\
\text { refused chemotherapy, } \\
\text { surgery, radiation } \\
\text { therapy }\end{array}$ & 2 \\
\hline
\end{tabular}

GE Medical Systems, Milwaukee, WI, USA). The patients were positioned such that the tumor was aligned with the transducer with acoustic coupling through a wetted gel pad molded to fit the patient's extremity. The patient was positioned to maximize acoustic access to the tumor while avoiding bones and nerves. The tumor was localized using multiplanar proton-density and T2-weighted MRI. The images were manually segmented to delineate the tumor contour and skin surface. Adjacent nerves were demarcated so that the system would plan treatment without energy passing through these areas. The initial treatment plan was produced by the MRgFUS software and set the number and size of sonications as well as the energy and duration of each sonication and the interval between sonications. The parameters of the treatment plan, in particular the energy, size, and angle of each sonication, were manually modified before and during treatment to minimize heating on the skin and near structures such as nerves and vessels. Sonication energies were adjusted to avoid cavitation and also based on the temperature-energy response of the tumor. Although cavitation is beneficial if you want to create high levels of heat in anatomic areas with little concern about precision or inadvertent heating of surrounding tissues, when the tissue begins to cavitate, the ablation volume is difficult to predict or control. We were often treating near skin, nerve, vessels, and muscles, and we wanted to control the treatment to minimize the risk of damage to those structures. Final alignment of the transducer was performed using low-energy sonications within the tumor. Heating was monitored in real time using proton resonant frequency shift MR thermometry. Immediately after sonications were completed, postcontrast images of the nonperfused volume of tumor were used to evaluate for treatment effect. After recovery from anesthesia, patients were discharged home on the day of treatment.

After the treatment, patients were allowed to weightbear as tolerated without limitation.

A postprocedure phone call was made the day after treatment and routine followup clinical examination was performed 2 weeks after treatment. Tumor response was assessed with interval MR scans and clinical examinations performed at approximately 6 weeks, 12 weeks, 24 weeks, 48 weeks, and 72-week intervals posttreatment. Patient 2 had followup at 16, 36, 48, and 60 weeks because of logistic issues related to transportation and patient availability unrelated to the MRgHIFU treatment time of 6 hours. Treatment-related adverse events were recorded. Total treatment time, the number of sonications, and the energy per sonication were recorded. Total tumor volumes and nonperfused (nonenhancing) volumes were measured before and after treatment. Tumor volumes were calculated by manually contouring the total and nonperfused areas on each imaging slice and summing the areas using Osirix (http://www.osirix-viewer.com) image analysis software [16]. Treatment-related adverse events and any additional treatments were recorded. The decision to stage a patient's treatment into multiple sessions was made if we thought a tumor would be best treated from multiple directions, eg, medial and lateral, which would require repositioning the patient or if staging was thought, in the treating physician's opinion, to be safer for the patient. Functional assessment was done by comparing the pretreatment 1993 Musculoskeletal Tumor Society score with the score at the latest followup [3].

Change in tumor size among the study population was reported as mean with a $95 \%$ confidence interval. 


\section{Results}

The ablation accuracy for the four cadaver extremities was $5 \mathrm{~mm}, 3 \mathrm{~mm}, 8 \mathrm{~mm}$, and $8 \mathrm{~mm}$. All ablations were grossly within the target volume defined by the plastic fiducials (Fig. 2).

After MRgFUS all patients except Patient 4 had a decrease in the total size and perfused volume of their tumors (Table 2). The mean decrease in tumor size among the study population was $36 \%$ (95\% confidence interval, 7\%-66\%) (Fig. 3). No patient received additional systemic or local treatment such as chemotherapy or radiation. Treatment-related adverse events, most of which were very minor and self-limited, occurred in five patients (Table 2). Patient 1 was incidentally noted to have edema surrounding the pancreas on a routine MR scan taken immediately after treatment. Her lipase was slightly elevated but she was asymptomatic and her lipase normalized within weeks. Patient 2, who had a recurrent popliteal fossa tumor previously treated with surgery and radiation that was encasing the peroneal nerve, developed complete peroneal nerve palsy on a preexisting partial nerve palsy related to previous treatment. At last followup he has partial nerve recovery. This patient also developed blisters along the margin of a scar from prior surgery and received oral antibiotics to treat a clinically suspected cellulitis that resolved without sequela. Patient 4 has experienced a slight increase in the total size and perfused volume of the tumor since her last treatment. At the most recent followup, no patient was taking scheduled narcotics or other analgesic for pain control.

\section{Discussion}

Treatments for soft tissue tumors of the extremities that either improve local control or decrease treatment-related morbidity are needed. MRgFUS is a noninvasive therapeutic modality that may be useful to treat extremity tumors, especially in situations in which standard treatments would be associated with unacceptable morbidity or are ineffective as is the case with desmoid fibromatosis. In the current study we found that MRgFUS can be used to accurately ablate a target volume of tissue in a cadaver extremity. Six patients with progressive fibromatosis were treated with MRgFUS and their respective tumors stopped growing or regressed. Those patients who previously were on chemotherapy did not receive any additional treatments. The decision to stop adjuvant treatments was made after multidisciplinary discussion including shared decisionmaking with the patient after thorough discussion of risks and benefits. All patients had similar reasons to stop chemotherapy, which included cessation of tumor growth,

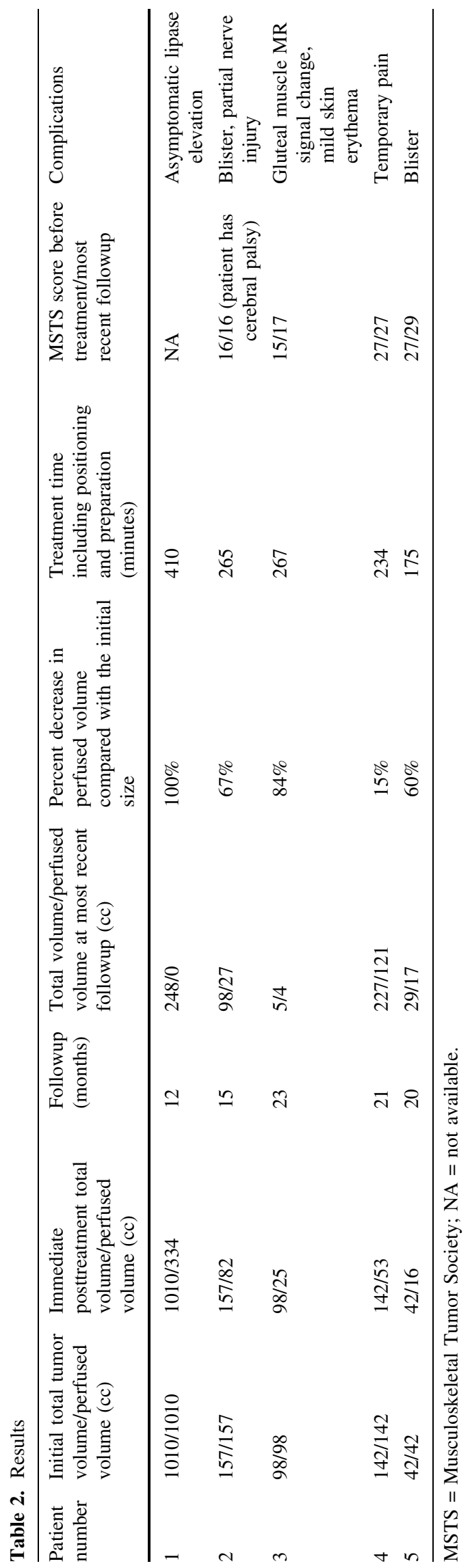



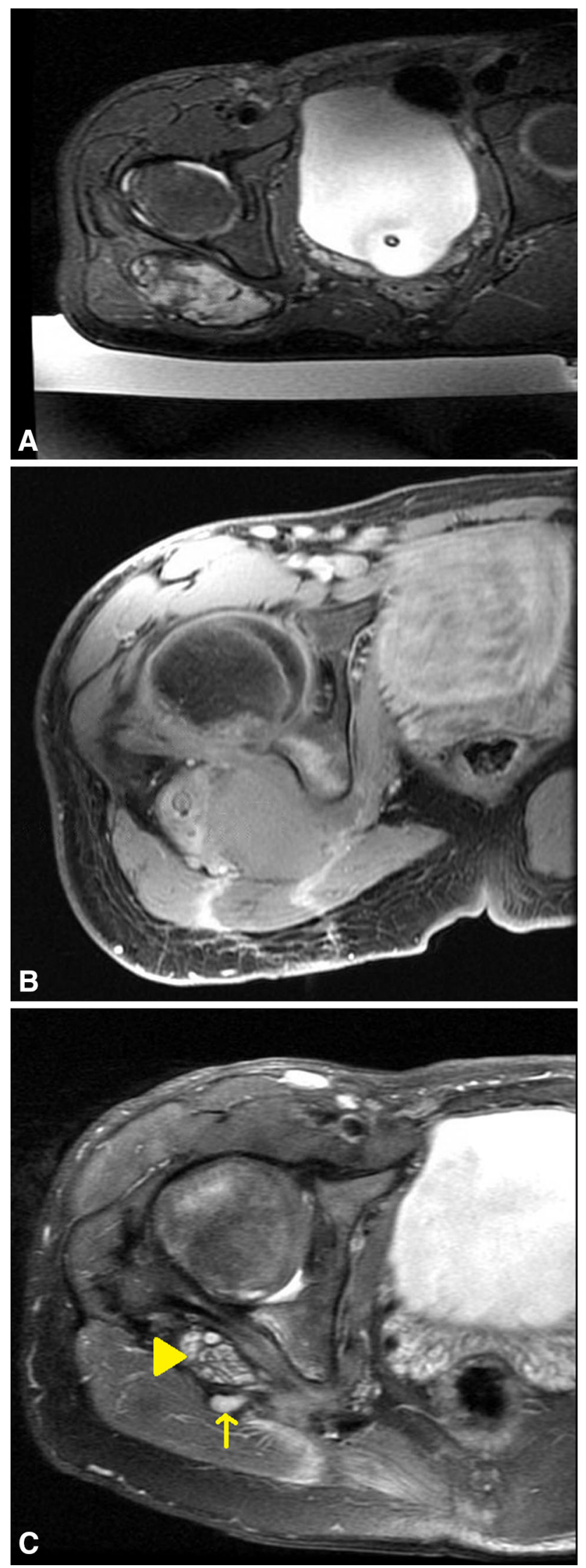

4 Fig. 3A-C A 15-year-old boy with (A) an axial T2-weighted MR image depicting a recurrent desmoid tumor in the buttock after aboveknee amputation; (B) axial T1 fat-suppressed postcontrast MR image showing absence of perfusion in tumor after MRgFUS treatment; and (C) axial T2-weighted MR image at 6 months followup demonstrating near complete tumor regression (arrow); the lobulated structure is the asymptomatic sciatic nerve stump (arrowhead). Signal change in bone is asymptomatic treatment-related edema that is decreasing on subsequent scans.

resolution of tumor-related symptoms, and desire to avoid chemotherapy-related side effects.

The study had a number of limitations. First, performing thermal ablation on nonperfused cadaver extremities, which do not have the same heating and cooling properties as living tissue, is not a precise simulation of in vivo conditions. Also to facilitate the experiments, target volumes were created as simple patterns rather than the more irregular complex shapes of real tumors. Therefore, translating thermal measurements and treatment dose maps from cadaver experience to human trials must be done with caution. We also note that gross assessment of tissue ablation is difficult to do and prone to error by the fact that cadaver muscle tissue is soft and mobile such that simple dissection may distort the shape of the region of interest. Postablation MRI assessment of accuracy is not possible in cadavers as a result of the inability to do contrast imaging. However, there were several goals of doing preliminary studies on cadavers including (1) practicing placement and alignment of extremities on the treatment device; (2) elucidating and resolving problems of coupling the extremity to the ultrasound transducer (what type of gel pad, drapes, amount of water to use, etc); and (3) determining if on a gross level we could successfully target and ablate a volume in the extremity, all of which we were able to accomplish and provided valuable insight into how to use MRgFUS safely and effectively. A second limitation of our study is the nature of our patient population, which was small and heterogeneous with respect to tumor location, size, and previous treatments. With the numbers available we cannot comment on how these variables affected outcome. However, as a preliminary report, the results of our study indicate that MRgFUS has potential to treat desmoid tumors and is worth continued investigation. The information in this study is based on a small number of patients with limited followup; longer term followup in more patients is needed to determine if MRgFUS will be a reliable and safe treatment for these patients.

This study provides preliminary information that MRguided high-intensity focused ultrasound can accurately target a simulated tumor volume. More extensive study with different shapes and locations of tumor simulations is necessary to confirm this, but we found reasonable 
accuracy in our planned targets. There are no other published studies to our knowledge that examine the use of MRgFUS on cadaver extremities.

Our results compare favorably with those of Wang et al, who reported on their experience using ultrasound-guided rather than MR-guided focused ultrasound, to treat 10 patients with extraabdominal desmoid tumors [19]. Although the technique reported in their study is different than the one we report here, the overall concept and mechanism of thermal ablation are similar. The mean age of their patients was 22 years and the mean tumor size was $9 \mathrm{~cm}$ in greatest dimension. Eight patients had recurrent tumors, whereas two patients had primary tumors. Two patients had multiple treatments. At a mean of 30 months followup, all tumors were smaller by greater than $50 \%$ compared with pretreatment. In our study the decrease in average tumor size was $35 \%$. A possible reason for this difference is that three of our patients had tumors in anatomic locations adjacent to neurovascular structures (two popliteal fossa tumors and one posterior ankle). We acknowledge this is a relatively new technology and there is undoubtedly a learning curve. Therefore, as a general treatment principle, we erred on the side of undertreating tumors to minimize side effects and emphasize patient safety, especially knowing that patients can have future treatments as needed without concern for cumulative dose effects. Patient 4 had a slight increase in the size of her tumor. This is likely the result of the fact that the tibial and peroneal nerves are partially encased in tumor, which precluded us from treating the entire tumor to avoid nerve injury. The treated portion of the tumor is slowly regressing and is nonperfused on followup MR imaging, but the untreated portion is slowly increasing in size resulting in an overall increase in total tumor volume. We have counseled her and her family regarding all treatment options including MRgFUS and conventional treatments; however, she has deferred on additional therapy in favor of observation because she is asymptomatic and feels well. Complications included first-degree burns and localized pain at the treatment site that resolved within 1 to 3 days after treatment. One patient with a preexisting partial peroneal nerve palsy developed complete palsy after MRgFUS treatment. Fortunately it improved with followup. No patient received adjuvant treatments and no patient had functional impairment as a result of treatment. $\mathrm{Hu}$ et al reported the successful use of ultrasound-guided high-intensity focused ultrasound to treat a multiply recurrent chest wall synovial sarcoma in a 51-year-old man [7]. At 2 years followup he is without evidence of local recurrence. To our knowledge there are no other reports of using high-intensity focused ultrasound to treat extremity soft tissue tumors. However, this technology is used for the treatment of bone tumors and although there are similarities between the techniques for treating bone versus soft tissue tumors, there are important differences [5, 14]. Most notable is that bone absorbs ultrasound to a much higher degree than soft tissue, making it technically easier and safer to deliver ablative energy to bone tumors. These differences need to be accounted for when comparing bone and soft tissue treatment results or study design.

The results of this study indicate that treatment with MRgHIFU led to regression in four of five patients with extraabdominal desmoid tumors. Although the small sample size prohibits drawing any definitive conclusions, we believe our experience provides evidence that MRgHIFU may be useful as a novel treatment modality for desmoid tumors and is worth further investigation. Further experience with MRgHIFU may show that it may be a treatment option for other extremity soft tissue tumors such as recurrent sarcomas and benign tumors. It might also serve as an adjuvant to existing treatment options for these tumors. Collaboration between orthopaedic oncologists and radiologists is important to define the treatment indications, goals, and outcome measures for MRgFUS.

Acknowledgments We thank Shannon Walters BS, RT, of the Stanford 3D radiology laboratory for assisting in MR image interpretation and calculating tumor volumes.

\section{References}

1. Ballo MT, Zagars GK, Pollack A, Pisters PW, Pollack RA. Desmoid tumor: prognostic factors and outcome after surgery, radiation therapy, or combined surgery and radiation therapy. $J$ Clin Oncol. 1999;17:158-167.

2. Boutrand J-P. Biocompatibility and Performance of Medical Devices. Cambridge, UK: Woodhead Publishing; 2012.

3. Enneking WF, Dunham W, Gebhardt MC, Malawar M, Pritchard DJ. A system for the functional evaluation of reconstructive procedures after surgical treatment of tumors of the musculoskeletal system. Clin Orthop Relat Res. 1993;286:241-246.

4. Fiore M, Rimareix F, Mariani L, Domont J, Collini P, Le Pechoux C, Casali PG, Le Cesne A, Gronchi A, Bonvalot S. Desmoid-type fibromatosis: a front-line conservative approach to select patients for surgical treatment. Ann Surg Oncol. 2009;16: 2587-2593.

5. Geiger D, Napoli A, Conchiglia A, Gregori LM, Arrigoni F, Bazzocchi A, Busacca M, Moreschini O, Mastantuono M, Albisinni U, Masciocchi C, Catalano C. MR-guided focused ultrasound (MRgFUS) ablation for the treatment of nonspinal osteoid osteoma: a prospective multicenter evaluation. J Bone Joint Surg Am. 2014;96:743-751.

6. Haar GT, Coussios C. High intensity focused ultrasound: physical principles and devices. Int J Hyperthermia. 2007;23:89-104.

7. Hu X, Cai H, Zhou M, He H, Tian W, Hu Y, Chen L, Deng Y. New clinical application of high-intensity focused ultrasound: local control of synovial sarcoma. World J Surg Oncol. 2013;11:265. 
8. Hynynen K. The feasibility of interstitial ultrasound hyperthermia. Med Phys. 1992;19:979-987.

9. Hynynen K, Damianou C, Darkazanli A, Unger E, Schenck JF. The feasibility of using MRI to monitor and guide noninvasive ultrasound surgery. Ultrasound Med Biol. 1993;19:91-92.

10. Lev D, Kotilingam D, Wei C, Ballo MT, Zagars GK, Pisters PW, Lazar AA, Patel SR, Benjamin RS, Pollock RE. Optimizing treatment of desmoid tumors. J Clin Oncol. 2007;25:1785-1791.

11. Lewis JJ, Boland PJ, Leung DH, Woodruff JM, Brennan MF. The enigma of desmoid tumors. Ann Surg. 1999;229:866-872; discussion 872-873.

12. Liberman B, Gianfelice D, Inbar Y, Beck A, Rabin T, Shabshin N, Chander G, Hengst S, Pfeffer R, Chechick A, Hanannel A, Dogadkin O, Catane R. Pain palliation in patients with bone metastases using MR-guided focused ultrasound surgery: a multicenter study. Ann Surg Oncol. 2009;16:140 146.

13. Mullen JT, Delaney TF, Kobayashi WK, Szymonifka J, Yeap BY, Chen YL, Rosenberg AE, Harmon DC, Choy E, Yoon SS, Raskin KA, Petur Nielsen G, Hornicek FJ. Desmoid tumor: analysis of prognostic factors and outcomes in a surgical series. Ann Surg Oncol. 2012;19:4028-4035.

14. Napoli A, Anzidei M, Marincola BC, Brachetti G, Ciolina F, Cartocci G, Marsecano C, Zaccagna F, Marchetti L, Cortesi E, Catalano C. Primary pain palliation and local tumor control in bone metastases treated with magnetic resonance-guided focused ultrasound. Invest Radiol. 2013;48:351-358.

15. Ren XL, Zhou XD, Zhang J, He GB, Han ZH, Zheng MJ, Li L, Yu M, Wang L. Extracorporeal ablation of uterine fibroids with high-intensity focused ultrasound: imaging and histopathologic evaluation. J Ultrasound Med. 2007;26:201-212.

16. Rosset A, Spadola L, Ratib O. OsiriX: an open-source software for navigating in multidimensional DICOM images. $J$ Digit Imaging. 2004;17:205-216.

17. Salas S, Dufresne A, Bui B, Blay JY, Terrier P, Ranchere-Vince D, Bonvalot S, Stoeckle E, Guillou L, Le Cesne A, Oberlin O, Brouste V, Coindre JM. Prognostic factors influencing progression-free survival determined from a series of sporadic desmoid tumors: a wait-and-see policy according to tumor presentation. J Clin Oncol. 2011;29:3553-3558.

18. Schlemmer M. Desmoid tumors and deep fibromatoses. Hematol Oncol Clin North Am. 2005;19:565-571, vii-viii.

19. Wang Y, Wang W, Tang J. Ultrasound-guided high intensity focused ultrasound treatment for extra-abdominal desmoid tumours: preliminary results. Int J Hyperthermia. 2011;27:648-653.

20. Zhang L, Chen WZ, Liu YJ, Hu X, Zhou K, Chen L, Peng S, Zhu H, Zou HL, Bai J, Wang ZB. Feasibility of magnetic resonance imaging-guided high intensity focused ultrasound therapy for ablating uterine fibroids in patients with bowel lies anterior to uterus. Eur J Radiol. 2010;73:396-403. 